\title{
Assessment of Global Voltage Stability Margin through Radial Basis Function Neural Network
}

\author{
Akash Saxena and Ankit Kumar Sharma \\ Department of Electrical Engineering, Swami Keshvanand Institute of Technology, Jaipur, India \\ Correspondence should be addressed to Akash Saxena; aakash.saxena@hotmail.com \\ Received 30 June 2016; Accepted 30 August 2016 \\ Academic Editor: Gorazd Stumberger
}

Copyright (C) 2016 A. Saxena and A. K. Sharma. This is an open access article distributed under the Creative Commons Attribution License, which permits unrestricted use, distribution, and reproduction in any medium, provided the original work is properly cited.

\begin{abstract}
Dynamic operating conditions along with contingencies often present formidable challenges to the power engineers. Decisions pertaining to the control strategies taken by the system operators at energy management centre are based on the information about the system's behavior. The application of ANN as a tool for voltage stability assessment is empirical because of its ability to do parallel data processing with high accuracy, fast response, and capability to model dynamic, nonlinear, and noisy data. This paper presents an effective methodology based on Radial Basis Function Neural Network (RBFN) to predict Global Voltage Stability Margin (GVSM), for any unseen loading condition of the system. GVSM is used to assess the overall voltage stability status of the power system. A comparative analysis of different topologies of ANN, namely, Feedforward Backprop (FFBP), Cascade Forward Backprop (CFB), Generalized Regression (GR), Layer Recurrent (LR), Nonlinear Autoregressive Exogenous (NARX), ELMAN Backprop, and Feedforward Distributed Time Delay Network (FFDTDN), is carried out on the basis of capability of the prediction of GVSM. The efficacy of RBFN is better than other networks, which is validated by taking the predictions of GVSM at different levels of Additive White Gaussian Noise (AWGN) in input features. The results obtained from ANNs are validated through the offline Newton Raphson (N-R) method. The proposed methodology is tested over IEEE 14-bus, IEEE 30-bus, and IEEE 118-bus test systems.
\end{abstract}

\section{Introduction}

In recent years, the power system stability issues are prominent and possess more relevance due to a competitive business environment. The existing generation and transmission utilities are working on their operating limits due to exponential increase in the load demand [1].

The burning issue with the modern power networks is to accommodate an escalating demand without an expansion of the transmission utilities. With this constraint, the voltage stability has emerged as a potential area of research. Two critical findings are carried out through stability studies. The first one is the assessment of the critical point of voltage stability and second area suggests preventive control through load shedding and generator rescheduling. IEEE CIGRE task force committee defined voltage stability as an ability of the power system to maintain acceptable and constant voltage level at all buses in the system under normal conditions and after being subjected to the disturbance $[1,2]$. Therefore, voltage stability analysis is necessary to identify the critical buses in a power system.

Voltage collapse is a phenomenon characterized by the gradual decrement in system voltage along with the system operating point. Often, the assessment of the voltage stability is carried out through the calculation of the stability indices [3-15]. Table 1 shows the comparative study of different indicators along with the limitations.

On the basis of literature survey, it can be concluded that the indicator for voltage stability assessment should possess the following qualities.

(a) There should be a simple correlation between the indicator and the system's controllable parameter.

(b) Corrective measures can be derived from the values of indicator.

With dynamic operating scenario, the prediction of voltage collapse through indicators is a slave of the performance 
TABLE 1: Comparative analysis of different voltage stability indicators.

\begin{tabular}{|c|c|c|}
\hline $\begin{array}{l}\text { Name of } \\
\text { indicator }\end{array}$ & Properties of indicator & Summary \\
\hline $\begin{array}{l}\text { GVSM [3], } \\
\text { L-index [4], } \\
\text { VCPI [5], } \\
\text { ENVCI [6], } \\
\text { VSI [7], } \\
\text { LCPI [8] } \\
\end{array}$ & $\begin{array}{l}\text { Based on Newton Raphson (NR) } \\
\text { load flow solution }\end{array}$ & $\begin{array}{l}\text { (1) The line indices are suitable for constant power load. The } \\
\text { results may be pessimistic for changed load profile. } \\
\text { (2) The convergence of NR algorithm is affected by the vicinity } \\
\text { of voltage collapse point. }\end{array}$ \\
\hline $\begin{array}{l}\text { References } \\
{[9,15]}\end{array}$ & $\begin{array}{l}\text { Index based on sensitivity } \\
\text { analysis }\end{array}$ & $\begin{array}{l}\text { (1) Sensitive based methods are computationally intensive and } \\
\text { required computation of derivatives. } \\
\text { (2) Dynamic operating conditions introduce errors in the } \\
\text { calculations of the derivatives. Moreover the correlation } \\
\text { between the voltage and reactive power should be continuous in } \\
\text { nature. }\end{array}$ \\
\hline $\begin{array}{l}\text { References } \\
{[10-14]}\end{array}$ & $\begin{array}{l}\text { These indices are based on static } \\
\text { and dynamic bifurcations }\end{array}$ & $\begin{array}{l}\text { (1) Bifurcations can be detected through the calculation of } \\
\text { eigenvalues. Eigenvalues are system specific and vulnerable to } \\
\text { topological changes. } \\
\text { (2) These indicators cannot be useful for loadability margin } \\
\text { evaluation and relative voltage stability determination. }\end{array}$ \\
\hline
\end{tabular}

of load flow routine and accuracy of the calculation of derivatives and Jacobian matrices. It is to be noted here that the prediction of the voltage stability under unseen operating condition or contingency cannot be efficiently executed within a safe time limit. Hence, the initiation of any preventive or emergency control strategy is not possible. With the development of the smart grid, efficient technologies are invited by the operators to participate in the process of operation and control of the power system. With this motivation, the paper presents an application of RBFN approach for online monitoring of voltage stability.

A network equivalence framework to predict the global scenario of voltage stability is developed by reducing the actual system into an equivalent two-bus system. The GVSM [3] is used for indicating the state of the actual system. All the parameters of the equivalent system are obtained from the load flow solution of the original system. This equivalent system is nothing but a power line having series equivalent impedance with a load at the receiving end, but the sending end voltage is kept at the reference voltage. The concept of single line equivalent is further used to determine the voltage collapse proximity.

In this paper, RBFN network of the ANN family is employed to predict the GVSM for various system operating loading. The proposed online scheme has the ability to get it adapted when subjected to any new and unseen operating condition.

This scheme is validated on standard IEEE 14-bus, 30-bus, and 118-bus power systems. The following are the research objectives of this manuscript:

(a) To present the mathematical framework of GVSM and calculate the GVSM for IEEE 14-bus, 30-bus, and 118-bus test system.

(b) To develop a supervised learning prediction engine with the help of offline simulation results to identify the GVSM for different system operating loading. (c) To present a meaningful comparison between different ANN topologies as a predictor and analyze the efficacy of the supervised learning prediction engine in the presence of AWGN.

The remaining part of the paper is presented as follows; in Sections 2 and 3 mathematical work of GVSM is presented. In Section 4, brief details of proposed RBFN are incorporated. In Section 5 simulation results are presented and finally in Section 6 the conclusion and future scope of the work are presented.

\section{Equivalent Two-Bus Pi-Network}

The equivalent two-bus pi-network model is developed as follows: Let us assume a two-bus equivalent network in which a generator bus is assumed as a sending end bus and a load bus is assumed as a receiving end bus as shown in Figure 1. The behavior and properties of the proposed two-bus equivalent model should be the same as the multibus network. Hence, this makes the evaluation of voltage stability possible [3]. Therefore, the power equations for the two-bus equivalent network can be written as

$$
\begin{aligned}
& S_{\mathrm{g}}=P_{\mathrm{g}}+j Q_{\mathrm{g}}=\vec{V}_{\mathrm{s}} \vec{I}_{\mathrm{s}}^{*}=\left(S_{\mathrm{se}}+S_{\mathrm{sh}}\right)+S_{\text {load }}, \\
& S_{\mathrm{se}}=\left(\vec{V}_{\mathrm{s}}-\vec{V}_{\mathrm{r}}\right) \vec{I}_{\mathrm{se}}^{*}, \\
& S_{\mathrm{sh}}=\vec{V}_{\mathrm{s}} \vec{I}_{\mathrm{shs}}^{*}+\vec{V}_{\mathrm{r}} \vec{I}_{\mathrm{shr}}^{*} .
\end{aligned}
$$

Applying KCL at node $m$ we get

$$
\vec{I}_{\mathrm{se}}^{*}=\frac{S_{\mathrm{g}}}{\vec{V}_{\mathrm{s}}}-S_{\mathrm{sh}}\left(\frac{\vec{V}_{\mathrm{s}}^{*}}{\left|\vec{V}_{\mathrm{s}}\right|^{2}+\left|\vec{V}_{\mathrm{r}}\right|^{2}}\right) .
$$




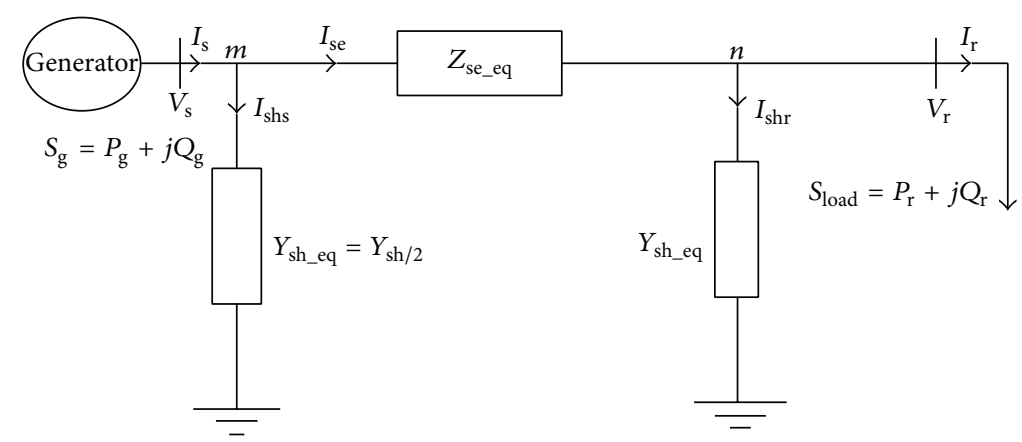

FIGURE 1: Two-bus pi-equivalent network.

Similarly at node $n$

$$
\vec{I}_{\mathrm{se}}^{*}=S_{\mathrm{sh}}\left(\frac{\vec{V}_{\mathrm{s}}^{*}}{\left|\vec{V}_{\mathrm{s}}\right|^{2}+\left|\vec{V}_{\mathrm{r}}\right|^{2}}\right)+\frac{S_{\mathrm{load}}}{\vec{V}_{\mathrm{r}}},
$$

where $V_{\mathrm{s}}, V_{\mathrm{r}}$, and $I_{\mathrm{s}}, I_{\mathrm{r}}$ are the sending and receiving end voltages and currents; $I_{\text {se }}$ is the current through series equivalent impedance; $I_{\mathrm{shs}}, I_{\mathrm{shr}}$ are the shunt branch currents at sending and receiving end, respectively.

After the calculations, we get the equivalent series impedance and equivalent shunt admittance.

$$
\begin{aligned}
& Z_{\text {se_eq }}=\frac{\left(\vec{V}_{\mathrm{s}}-\vec{V}_{\mathrm{r}}\right)}{\vec{I}_{\mathrm{se}}}, \\
& Y_{\text {sh_eq }}=\frac{\vec{I}_{\mathrm{shr}}}{\vec{V}_{\mathrm{r}}}=\frac{\vec{I}_{\mathrm{shs}}}{\vec{V}_{\mathrm{s}}} .
\end{aligned}
$$

This equivalent two-bus pi-network is used for obtaining the GVSM.

\section{Global Voltage Stability Analysis of Multibus Power System}

When the two-bus network equivalent to a multibus power system is obtained, the global voltage stability index can be formulated in a straightforward manner from the parameters of the global network as follows.

Here the voltage-current relation in terms of $\mathrm{ABCD}$ parameters for pi-equivalent two-bus circuit of the transmission line is given by

$$
\left[\begin{array}{c}
V_{\mathrm{s}} \\
I_{\mathrm{s}}
\end{array}\right]=\left[\begin{array}{ll}
A & B \\
C & D
\end{array}\right]\left[\begin{array}{l}
V_{\mathrm{r}} \\
I_{\mathrm{r}}
\end{array}\right],
$$

where

$$
\begin{aligned}
& A=D=1+\frac{Y Z}{2} ; \\
& B=Z ; \\
& C=Y\left(1+\frac{Y Z}{4}\right) .
\end{aligned}
$$

Assume

$$
\left[Z=Z_{\text {se_eq }}, \frac{Y}{2}=Y_{\text {sh_eq }}\right]
$$

Let us assume

$$
\begin{aligned}
A & =|A| \angle \alpha ; \\
B & =|B| \angle \beta ; \\
\vec{V}_{\mathrm{s}} & =\left|\vec{V}_{\mathrm{s}}\right| \angle \theta ; \\
\vec{V}_{\mathrm{r}} & =\left|\vec{V}_{\mathrm{r}}\right| \angle \delta ; \quad \delta<\theta .
\end{aligned}
$$

Solving for the receiving end current:

$$
I_{\mathrm{r}}=\frac{\left|\vec{V}_{\mathrm{s}}\right|}{|B|} \angle \theta-\beta-\frac{|A|\left|\vec{V}_{\mathrm{r}}\right|}{|B|} \angle \alpha-\beta+\delta .
$$

Complex power of receiving end is given by

$$
\begin{aligned}
& S_{\mathrm{r}}=\vec{V}_{\mathrm{r}} \vec{I}_{\mathrm{r}}^{*} \\
& \quad=\left|\vec{V}_{\mathrm{r}}\right| \angle \delta\left[\frac{\left|\vec{V}_{\mathrm{s}}\right|}{|B|} \angle-\theta+\beta-\frac{|A|\left|\vec{V}_{\mathrm{r}}\right|}{|B|} \angle-\alpha+\beta-\delta\right] .
\end{aligned}
$$

Sending end voltage is constant; then the active and reactive power at the receiving end is given by

$$
\begin{aligned}
& P_{\mathrm{r}}=\frac{\left|\vec{V}_{\mathrm{r}}\right|}{|B|} \cos (\beta+\delta)-\frac{|A|\left|\vec{V}_{\mathrm{r}}\right|^{2}}{|B|} \cos (\beta-\alpha), \\
& Q_{\mathrm{r}}=\frac{\left|\vec{V}_{\mathrm{r}}\right|}{|B|} \sin (\beta+\delta)-\frac{|A|\left|\vec{V}_{\mathrm{r}}\right|^{2}}{|B|} \sin (\beta-\alpha) .
\end{aligned}
$$

The Jacobian matrix is given by

$$
\begin{aligned}
J= & {\left[\begin{array}{ll}
\frac{\partial P_{\mathrm{r}}}{\partial \delta} & \frac{\partial P_{\mathrm{r}}}{\partial V_{\mathrm{r}}} \\
\frac{\partial Q_{\mathrm{r}}}{\partial \delta} & \frac{\partial Q_{\mathrm{r}}}{\partial V_{\mathrm{r}}}
\end{array}\right]=\frac{1}{|B|} } \\
& \cdot\left[\begin{array}{c}
-\left|\vec{V}_{\mathrm{r}}\right| \sin (\beta+\delta) \cos (\beta+\delta)-2|A|\left|\vec{V}_{\mathrm{r}}\right| \cos (\beta-\alpha) \\
\left|\vec{V}_{\mathrm{r}}\right| \cos (\beta+\delta) \sin (\beta+\delta)-2|A|\left|\vec{V}_{\mathrm{r}}\right| \sin (\beta-\alpha)
\end{array}\right] .
\end{aligned}
$$




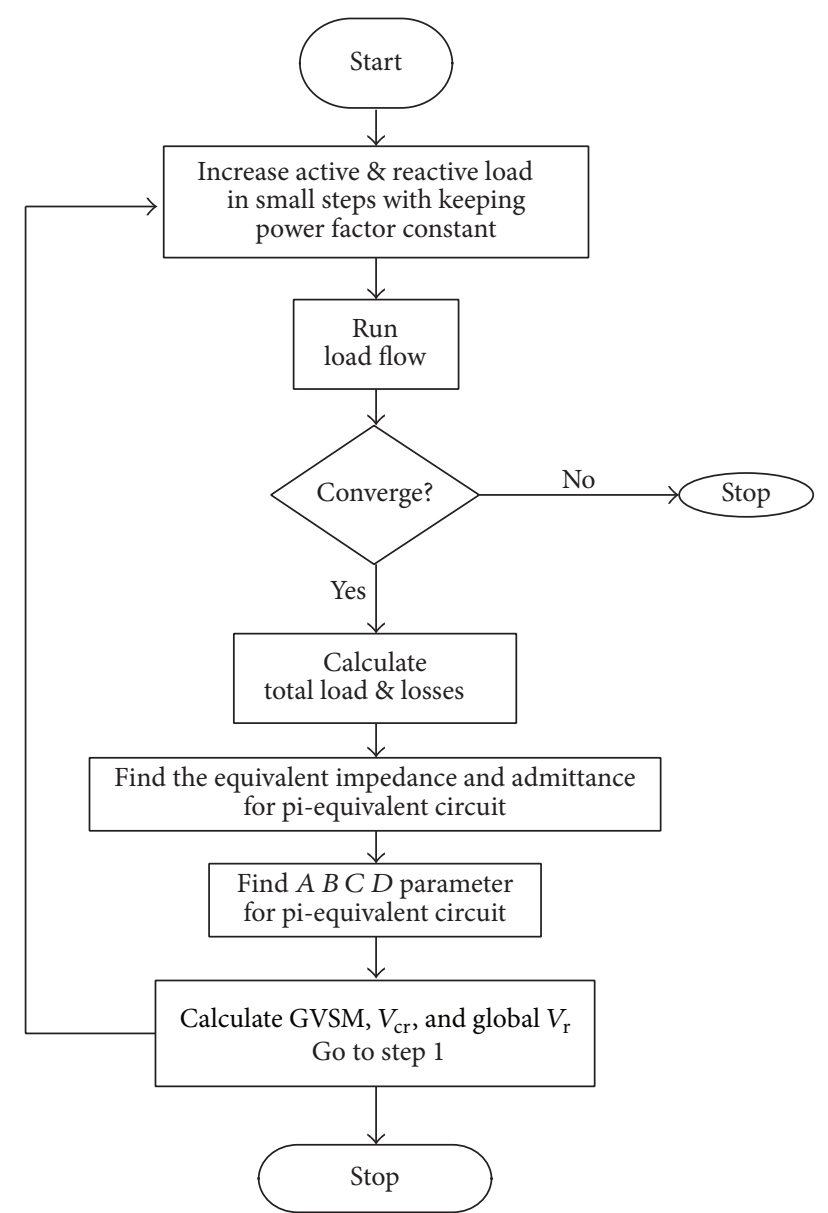

Figure 2: Algorithm to compute GVSM, $V_{\text {cr }}$, and global $V_{\mathrm{r}}$.

The determinant of Jacobian matrix is given in

$$
\Delta[J]=\frac{1}{|B|^{2}}\left[2|A|\left|\vec{V}_{\mathrm{r}}\right|^{2} \cos (\delta+\alpha)-\left|\vec{V}_{\mathrm{r}}\right|\right] .
$$

At the critical point of voltage stability, critical voltage index $(\mathrm{CVI})$ is given in

$$
\left|\vec{V}_{\mathrm{r}}\right|=V_{\mathrm{cr}}=\frac{1}{2|A| \cos (\delta+\alpha)} .
$$

Here $V_{\mathrm{cr}}$ is the critical value of the receiving end voltage at voltage stability. Low value of $V_{\text {cr }}$ indicates the system will have better voltage profile along with higher load handling capacity. To maintain global voltage stability, this condition should be satisfied: $\Delta[J]=0$. Therefore to secure global voltage stability, the GVSM can be defined as GVSM = $\Delta[J]$, given in (13). It indicates how far the present operating condition is from global system voltage collapse $[3,16]$. Figure 2 shows the flow of algorithm for computation of GVSM and CVI.

\section{RBF Neural Network Architecture}

The RBFN is a feedforward neural network, which consists of an input layer, one hidden layer, and one output layer.
The value of neurons of the input layer feeds in the hidden layer, a hidden layer which holds each neuron with radial basis activation function, and an output layer which holds each neuron with a linear activation function. The initiating centre, width for RBF units, and computing weights for connecters are combined to make a learning process for RBF neural network [17]. The idea about RBFN comes out from the theory of function approximation. According to this theory, there are two layers of feedforward network and a set of radial basis functions implemented by hidden node. The Gaussian function is normally used in it. The linear summation function as in a Multilayer Perceptron (MLP) is implemented by the output nodes. The network training is divided into two stages, in the first stage, weights are determined from input to hidden layer, and in the second stage weights are determined from hidden layer to output layer. This makes interpolation very effective.

For the training of ANN, the input data sets are generated from offline N-R load flow analysis by varying both real and reactive loads at all the buses randomly of their base case value. In data collection, the input data are divided into three categories, namely, train data, validation data, and test data. NR load flow analysis is conducted at all steps and corresponding GVSM is calculated. The real and reactive power loads on buses are considered as input features for building up the supervised learning models. Total 236 inputs for IEEE 118-bus system, 60 inputs for IEEE 30-bus system, and 28 inputs for IEEE 14-bus system are taken. By NR method GVSM of each line is obtained and the minimum values out of 358 for IEEE 118-bus system, out of 82 for IEEE 30-bus systems, and out of 40 for IEEE 14-bus systems are taken as output. Total 1000 samples are also generated by offline N-R load flow analysis method. $70 \%$ data of the samples are used for training, $20 \%$ data for validation, and $10 \%$ data for testing.

\section{Simulation Results}

A computer software programme has been developed in the MATLAB 2015b [18] environment to perform the simulations and run on a Pentium IV CPU, 2.69 GHz, and 1.84 GB RAM computer. To demonstrate the effectiveness of the proposed technique, IEEE 14-bus test system, IEEE 30-bus test system, and IEEE 118-bus test system have been used. IEEE 14-bus system represents a portion of the American Electric Power System which is located in the Midwestern US since February 1962. Basically, this 14-bus system has 14 buses, 5 generators, and 9 load buses. IEEE 30-bus system represents a portion of the American Electric Power System (in the Midwestern US) since December 1961. This system has 30 buses, 6 generators, and 24 load buses. IEEE 118-bus system has 118 buses, 51 generators, and 67 load buses [19].

5.1. Case Study of IEEE 14-Bus Test System. To validate the proposed approach, three operating scenarios are considered.

Case 1. It is near the base case (where the load buses are having the nominal values of real and reactive power loading). 


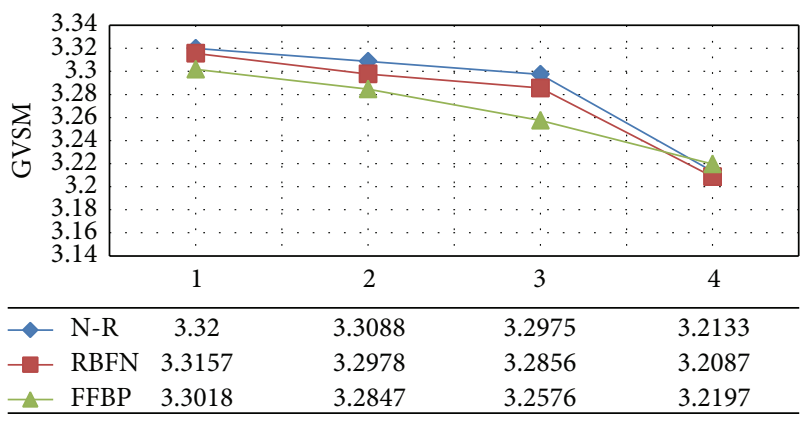

Figure 3: GVSM for operating Case 1.

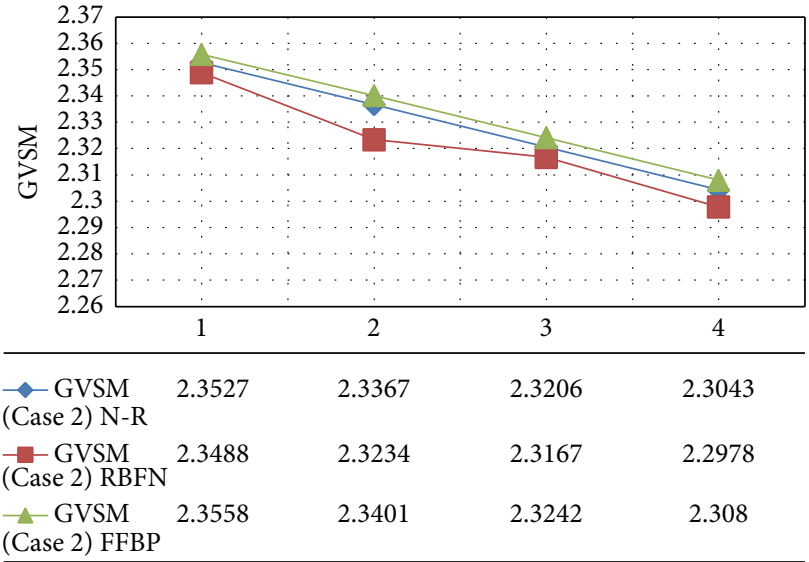

FIGURE 4: GVSM for operating Case 2.

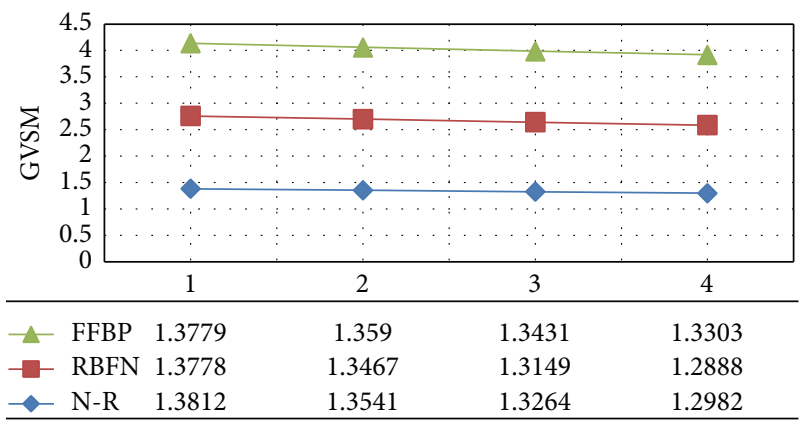

FIGURE 5: GVSM for operating Case 3.

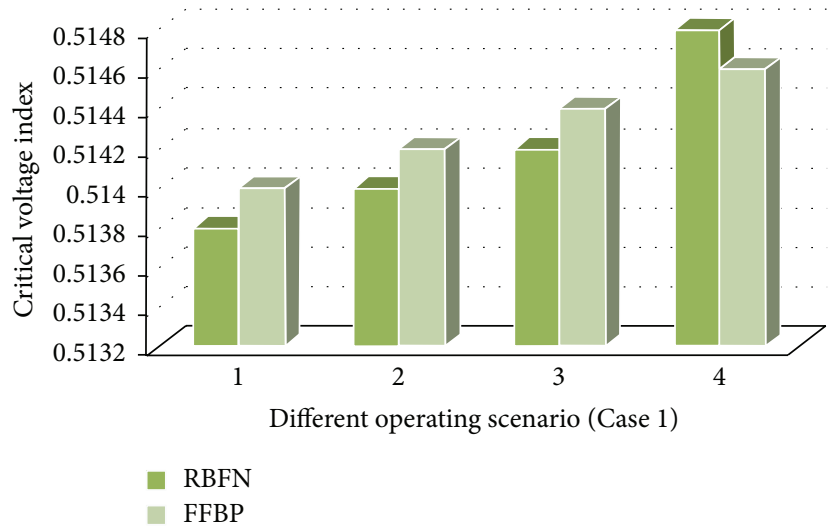

Figure 6: CVI for Case 1.

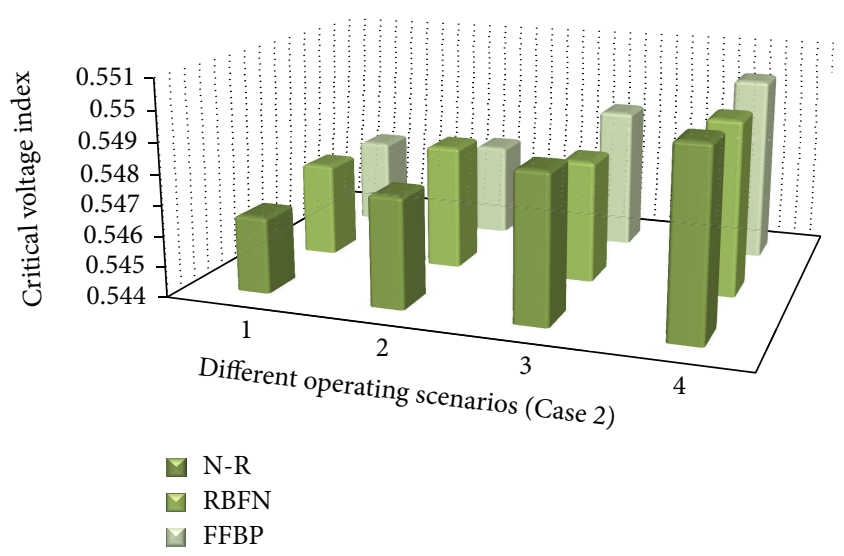

Figure 7: CVI for Case 2.

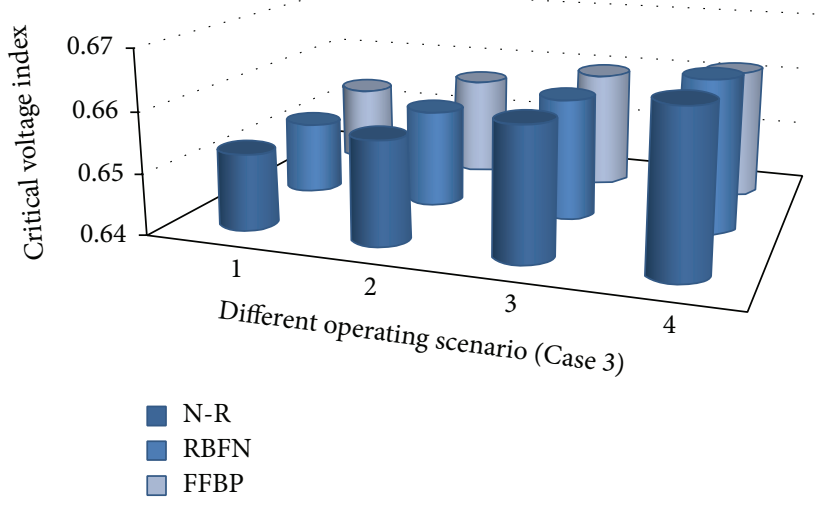

FIGURE 8: CVI for Case 3.

Case 2. It is medium load (increase of the system operating load by 2.24 p.u. from base case).

Case 3. It is high system operating load (increase of the system operating load by 3.2 p.u. from base case).

Figures 3-8 show the GVSM and CVI for IEEE 14-bus test system for three operating scenarios. Different intermediate loading conditions are incorporated to show the prediction efficacy of the supervised learning models. For each scenario four intermediate random loading conditions are considered and shown on the $x$-axis of Figures $3-8$. Due to space limitations detailed values of Jacobian matrices and CVIs are provided in the form of Supplementary Material available online at http://dx.doi.org/10.1155/2016/4858431 .

For Case 1, it is observed that the values of GVSM and CVI predicted by FFBP and RBFN fall in a secure range. In other words, near the base case the value of GVSM is higher for the system and the values of CVIs are lower. These values are validated by the offline N-R method. It is also observed that the prediction accuracy of RBFN is higher than FFBP. In Case 2, a considerable amount of decrease in numerical values of GVSM and increase in the CVI are observed by all prediction methods. For Case 3, after a continuous increase in the system operating load, the system has approached the point of collapse. The values of GVSM possess a decreasing 


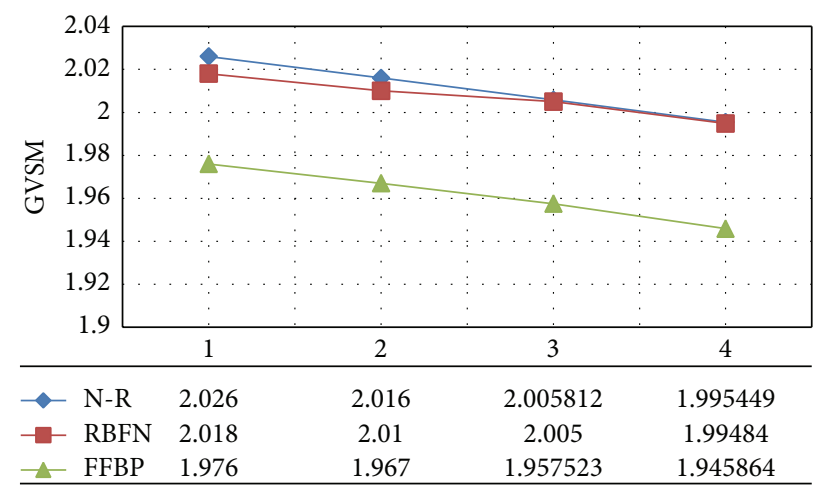

FIGURE 9: GVSM for operating Case 1.

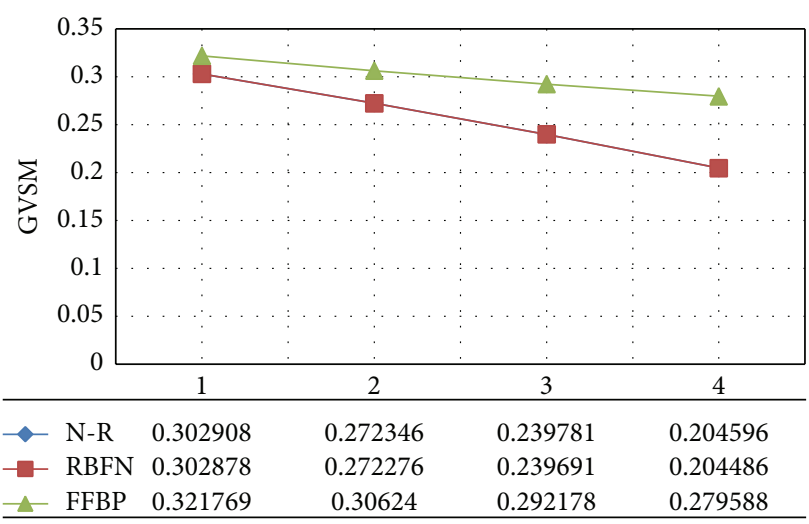

Figure 10: GVSM for operating Case 2.

tend and CVI becomes higher. The prediction capability of all the networks is verified by the offline N-R method. From this analysis, it is concluded that RBFN is a suitable topology to identify the critical buses in IEEE 14-bus test system.

Table 2 gives the GVSM at unknown system operating loading incorporating AWGN of different signal-to-noise ratios (SNRs). Presence of AWGN is a close replica of measurement errors and presence of harmonic loads and electronic measurement devices. At SNR 0.5 and 0.05, for unknown loading, the efficacy of RBFN is better than other networks, as can be observed in Table 2. RBFN and CFB topologies of ANN give the better results. Apart from this, the results from the FFBP networks, GR networks, LR networks, NARX networks, ELMAN, and FFDTD networks are satisfactory.

5.2. Case Study of IEEE 30-Bus Test System. To validate the proposed approach three operating scenarios are considered.

Case 1. It is near the base case (where the load buses are having the nominal values of real and reactive power loading).

Case 2. It is medium operating load (increase of the system operating load by 1.89 p.u.).

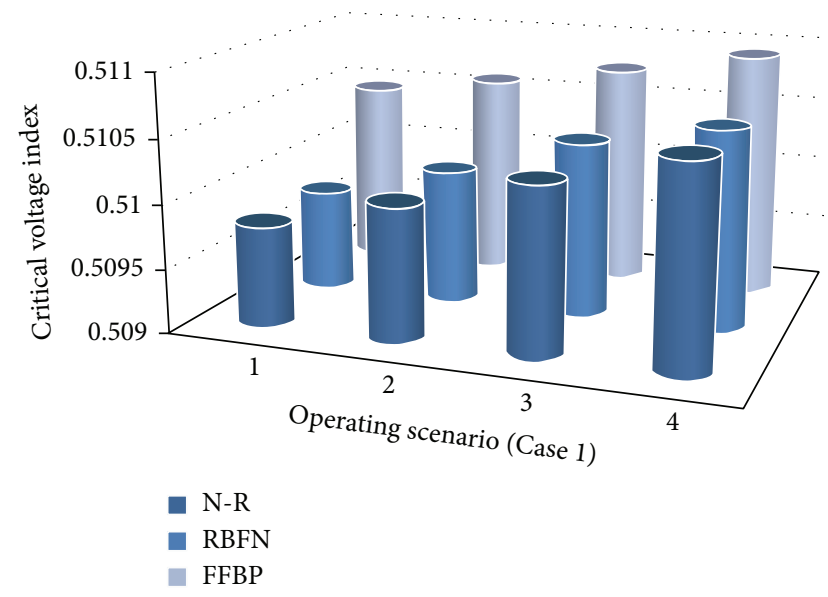

Figure 11: CVI for Case 1.

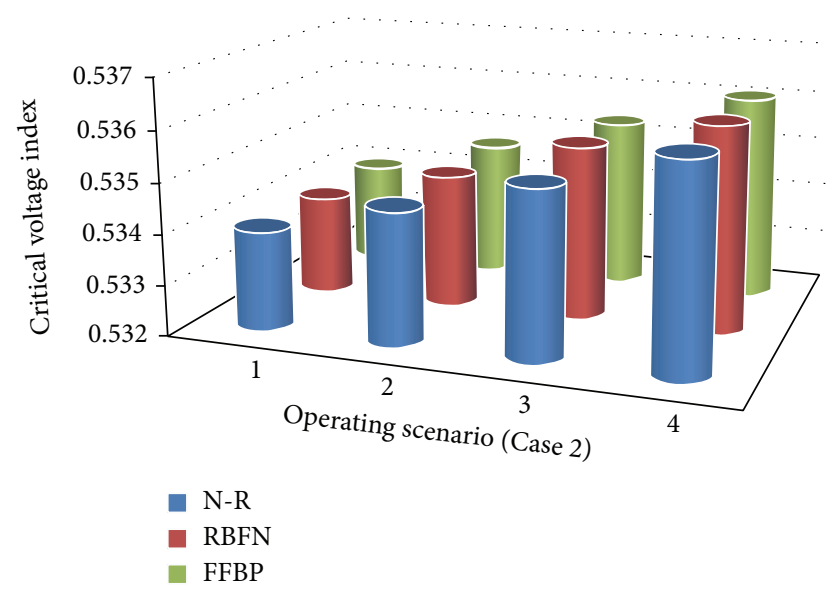

Figure 12: CVI for Case 2.

Case 3. It is high system operating load (increase of the system operating load by 2.7 p.u. from base case).

Figures 9-13 give the GVSM and CVI for the abovementioned operating cases.

It is observed that the values of GVSM and CVI predicted by FFBP and RBFN fall in a close range and the predictions are validated by the offline N-R method. It is observed that the prediction accuracy of RBFN is higher. For each scenario four intermediate random loading conditions are considered and shown on the $x$-axis of Figures 9-13. In Case 3, after a continuous increase in the system operating load the system has reached near the point of collapse. The values of GVSM tend to zero NR (0.204596), FFBP (0.279588), and RBFN (0.204486). From this analysis it is concluded that RBFN is a suitable topology to identify the critical buses in IEEE 30bus test system.

In Table 3, different unseen operating loading conditions are simulated and it is observed that the value of GVSM given by RBFN is the nearest value of GVSM given by the N-R offline method as compared to other networks. 


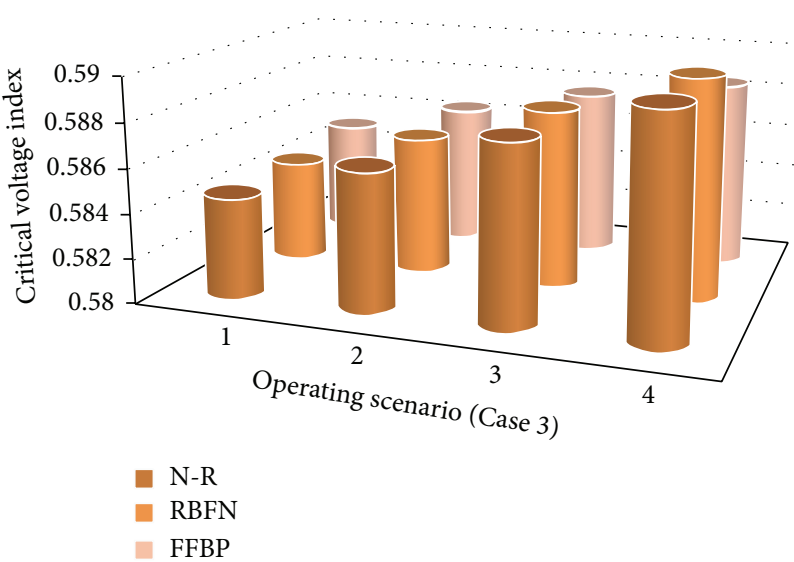

Figure 13: CVI for Case 3.

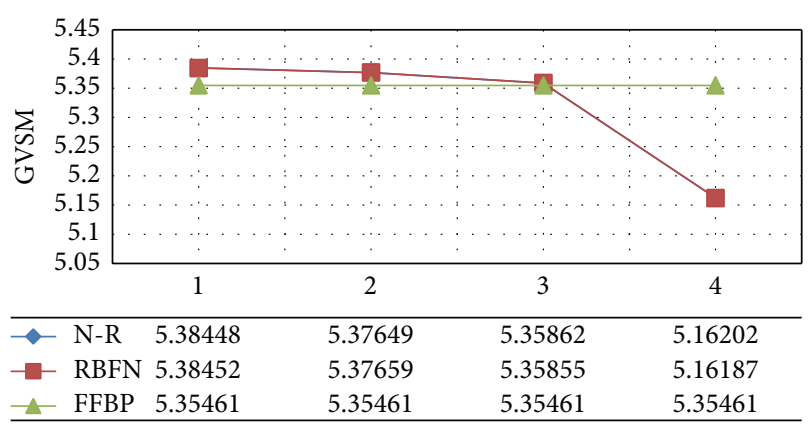

FIgURE 14: GVSM for operating Case 1.

5.3. Case Study of IEEE 118-Bus Test System. To validate the proposed approach three operating scenarios are considered.

Case 1. It is near the base case (where the load buses are having the nominal values of real and reactive power loading).

Case 2. It is medium load (increase of the system operating load by 0.90 p.u. from base case); that is, it is half of the high system operating loading.

Case 3. It is high system operating load (increase of the system operating load by 1.77 p.u. from base case).

Figures 14-18 give the GVSM and CVI for the abovementioned three cases.

As previously observed, the prediction capability of RBFN is better in comparison to FFBP. The same can be verified through the offline NR simulation results. For each scenario four intermediate random loading conditions are considered and shown on the $x$-axis of Figures $14-18$. It is interesting to observe that with the increment of the load, that is, for Case 3, the system has collapsed. Hence, it is giving the negative values of GVSM.

Table 4 gives the GVSM at unknown system operating load incorporating normal as well as noisy operating conditions. At SNR 0.5 and 0.05, for unknown loading, the efficacy of RBFN is better than other networks. The increment of the

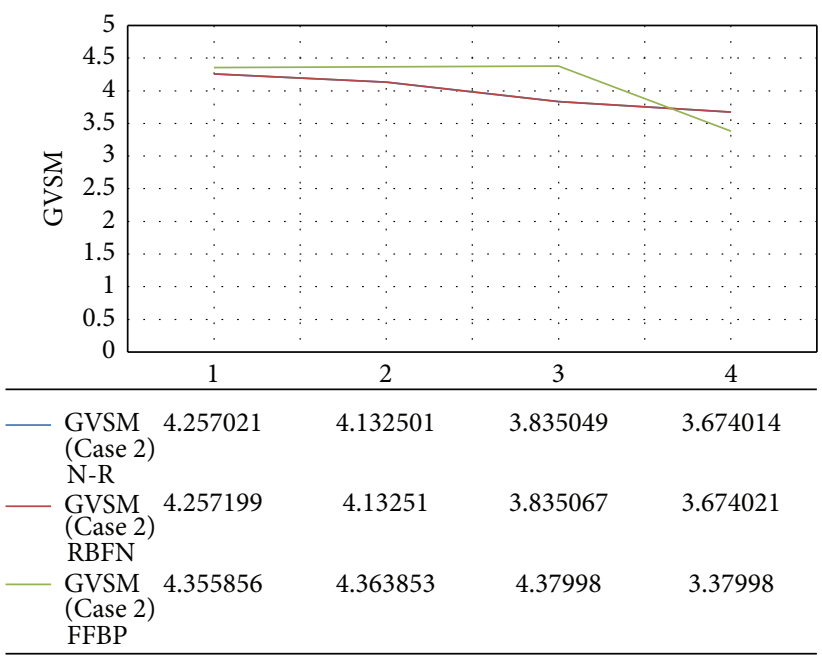

FIGURE 15: GVSM for operating Case 2.

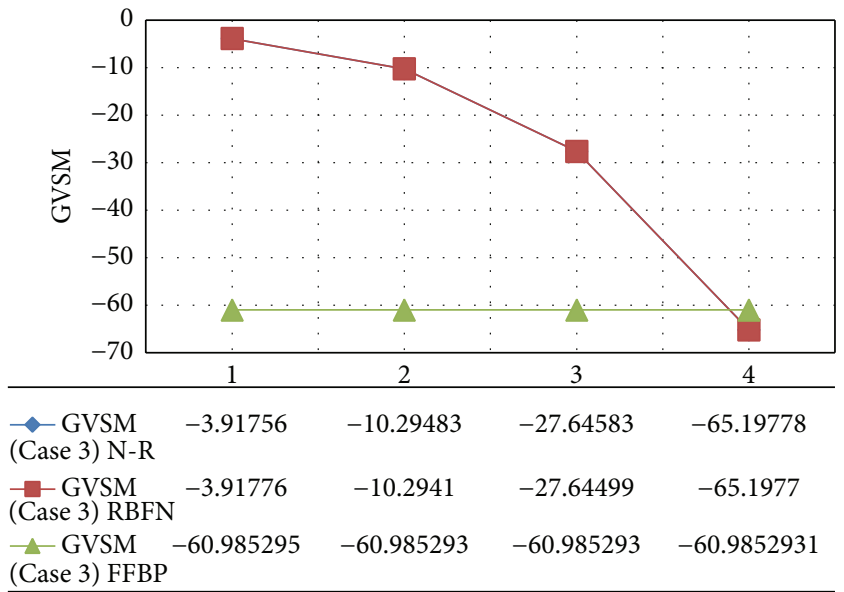

FIgURE 16: GVSM for operating Case 3.

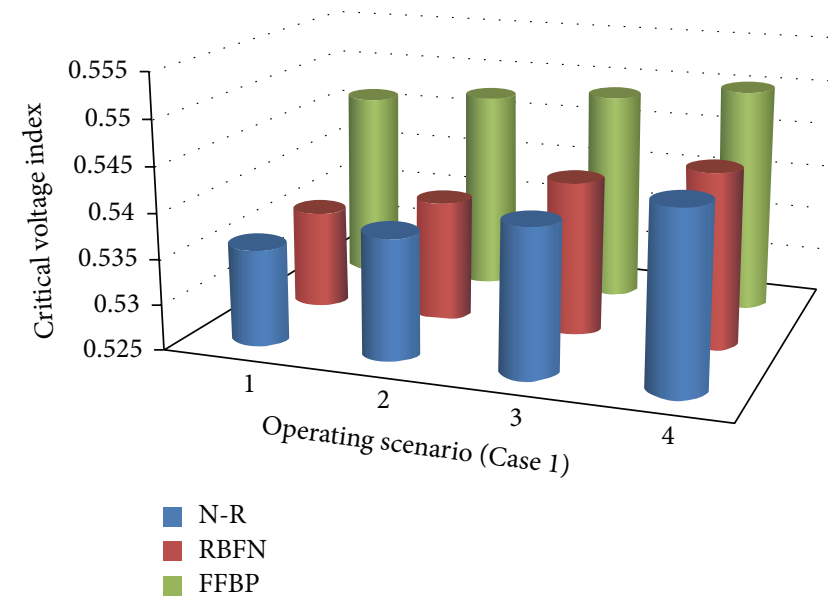

FIGURE 17: CVI for operating Case 1. 
TABLE 2: GVSM for IEEE 14-bus test system at unknown different operating loading incorporating noise.

\begin{tabular}{|c|c|c|c|c|c|c|c|c|c|}
\hline \multicolumn{10}{|c|}{ Value of GVSM for unknown loading at normal case } \\
\hline & NR & FFBP & CFB & GR & LR & NARX & RBFN & ELMAN & FFDTD \\
\hline $\begin{array}{l}\text { Operating load } \\
\text { (1.149 p.u.) }\end{array}$ & 3.2212 & 1.6369 & 3.0838 & 3.236 & 2.478 & 2.0081 & 3.2252 & 2.5647 & 2.5566 \\
\hline $\begin{array}{l}\text { Operating load } \\
1.339\end{array}$ & 2.8993 & 1.6275 & 3.0199 & 2.9019 & 2.7372 & 2.0781 & 2.8991 & 2.5638 & 2.5299 \\
\hline $\begin{array}{l}\text { Operating load } \\
2.2315\end{array}$ & 2.2657 & 2.6635 & 2.1953 & 2.2657 & 3.2673 & 2.2493 & 2.2659 & 2.4685 & 2.3028 \\
\hline $\begin{array}{l}\text { Operating load } \\
2.4951\end{array}$ & 2.0474 & 3.0925 & 1.7732 & 2.0474 & 3.2687 & 2.2684 & 2.0476 & 2.4184 & 2.2467 \\
\hline $\begin{array}{l}\text { Operating load } \\
3.1591\end{array}$ & 1.3522 & 3.203 & 1.3951 & 1.3521 & 3.1603 & 2.1735 & 1.3527 & 2.3458 & 2.1562 \\
\hline \multicolumn{10}{|c|}{ Value of GVSM for unknown loading at signal-to-noise ratio $0.5 \mathrm{db}$} \\
\hline & NR & FFBP & $\mathrm{CFB}$ & GR & LR & NARX & RBFN & ELMAN & FFDTD \\
\hline $\begin{array}{l}\text { Operating load } \\
1.149\end{array}$ & 3.2212 & 1.6077 & 3.1262 & 3.2315 & 2.3469 & 1.9414 & 3.211 & 2.5832 & 2.4903 \\
\hline $\begin{array}{l}\text { Operating load } \\
1.339\end{array}$ & 2.8993 & 1.6576 & 2.9979 & 2.898 & 2.7348 & 2.206 & 2.8989 & 2.3297 & 2.6217 \\
\hline $\begin{array}{l}\text { Operating load } \\
2.2315\end{array}$ & 2.2657 & 2.5865 & 2.158 & 2.2754 & 3.2403 & 2.1797 & 2.265 & 2.917 & 2.2091 \\
\hline $\begin{array}{l}\text { Operating load } \\
2.4951\end{array}$ & 2.0474 & 3.1172 & 1.8 & 2.0411 & 3.2453 & 2.2748 & 2.047 & 2.4592 & 2.2027 \\
\hline $\begin{array}{l}\text { Operating load } \\
3.1591\end{array}$ & 1.3522 & 3.1185 & 1.3689 & 1.342 & 3.1761 & 2.1339 & 1.3492 & 2.0972 & 2.159 \\
\hline \multicolumn{10}{|c|}{ Value of GVSM for unknown loading at signal-to-noise ratio $0.05 \mathrm{db}$} \\
\hline & NR & FFBP & CFB & GR & LR & NARX & RBFN & ELMAN & FFDTD \\
\hline $\begin{array}{l}\text { Operating load } \\
1.149\end{array}$ & 3.2212 & 1.4873 & 3.0989 & 3.23 & 2.644 & 1.8481 & 3.1988 & 2.6125 & 2.6644 \\
\hline $\begin{array}{l}\text { Operating load } \\
1.339\end{array}$ & 2.8993 & 1.7963 & 3.1318 & 2.9034 & 2.686 & 2.6166 & 2.8879 & 1.9629 & 2.6516 \\
\hline $\begin{array}{l}\text { Operating load } \\
2.2315\end{array}$ & 2.2657 & 2.4508 & 2.6347 & 2.2567 & 3.2917 & 2.2748 & 2.2552 & 2.3478 & 2.3044 \\
\hline $\begin{array}{l}\text { Operating load } \\
2.4951\end{array}$ & 2.0474 & 3.0864 & 1.8855 & 2.0361 & 3.2661 & 2.3683 & 2.011 & 1.819 & 2.3264 \\
\hline $\begin{array}{l}\text { Operating load } \\
3.1591\end{array}$ & 1.3522 & 3.1284 & 1.3836 & 1.379 & 3.1559 & 1.9611 & 1.3349 & 2.3348 & 2.1616 \\
\hline
\end{tabular}

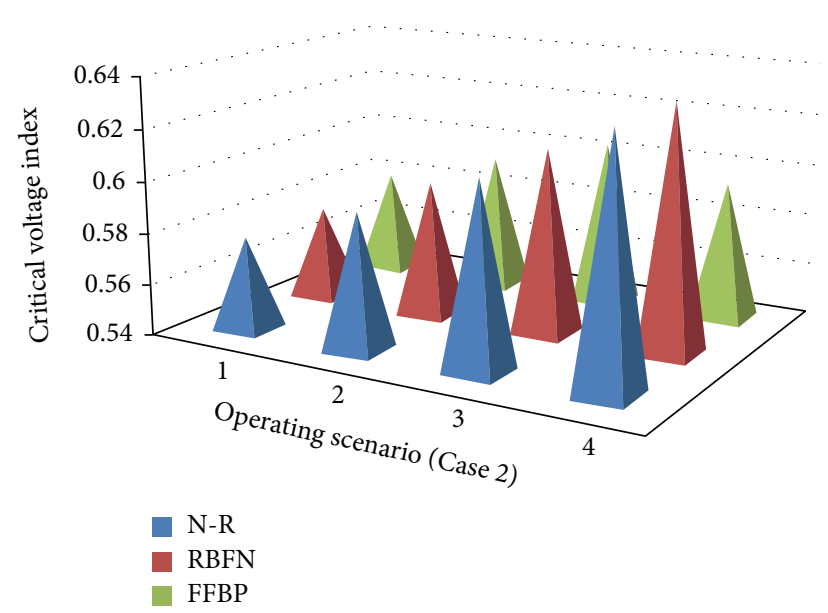

FIGURE 18: CVI for operating Case 2. system load in small and equidistant steps makes the value of GVSM lower with every step. Negative values of GVSM are the indication of collapse. The simulation results of the voltage stability analysis using proposed technique give better accuracy and reliability.

\section{Discussions}

(1) It is empirical to judge that the value of CVI is minimum, when $\delta+\alpha \approx 0$. The value of $A$ is one for transmission networks. From this it can be concluded that the minimum value of CVI is equal to 0.5 . With the increment in system load, increment in the values of CVI will be observed. For IEEE 14-bus system the CVI observed for Case 3 scenario 4 reached up to 0.66 . The high values of CVI are an indication of stress on lines. Similar patterns are observed for IEEE 30-bus and IEEE 118-bus system. Gradual increase in 
TABLE 3: GVSM for IEEE 30-bus test system at unknown different operating loading incorporating noise.

\begin{tabular}{|c|c|c|c|c|c|c|c|c|c|}
\hline \multicolumn{10}{|c|}{ Value of GVSM for unknown loading at normal case } \\
\hline & NR & FFBP & CFB & GR & LR & NARX & RBFN & ELMAN & FFDTD \\
\hline $\begin{array}{l}\text { Operating load } \\
1.1628\end{array}$ & 1.9862 & 1.9593 & 2.0219 & 1.9742 & 0.7938 & 1.4445 & 1.9849 & 0.4502 & 1.7873 \\
\hline $\begin{array}{l}\text { Operating load } \\
1.2118\end{array}$ & 1.8617 & 1.9432 & 2.0203 & 1.8625 & 0.8713 & 1.4237 & 1.8611 & 0.4794 & 1.7916 \\
\hline $\begin{array}{l}\text { Operating load } \\
1.5122\end{array}$ & 1.4953 & 1.7104 & 1.9924 & 1.4959 & 1.5513 & 1.2909 & 1.4951 & 0.587 & 1.8094 \\
\hline $\begin{array}{l}\text { Operating load } \\
1.8112\end{array}$ & 1.2351 & 1.3424 & 1.8337 & 1.2351 & 1.9035 & 1.1392 & 1.235 & 1.6006 & 1.8063 \\
\hline $\begin{array}{l}\text { Operating load } \\
2.5122\end{array}$ & 0.5201 & 0.6106 & 0.2401 & 0.52 & 1.9453 & 0.6958 & 0.5203 & 1.9932 & 1.7387 \\
\hline \multicolumn{10}{|c|}{ Value of GVSM for unknown loading at signal-to-noise ratio $0.5 \mathrm{db}$} \\
\hline & NR & FFBP & CFB & GR & LR & NARX & RBFN & ELMAN & FFDTD \\
\hline $\begin{array}{l}\text { Operating load } \\
1.1628\end{array}$ & 1.9862 & 1.9654 & 2.0249 & 1.9539 & 0.6874 & 1.4587 & 1.981 & 0.3749 & 1.7461 \\
\hline $\begin{array}{l}\text { Operating load } \\
1.2118\end{array}$ & 1.8617 & 1.834 & 2.0215 & 1.8579 & 0.3972 & 1.6843 & 1.8588 & 0.4354 & 1.7279 \\
\hline $\begin{array}{l}\text { Operating load } \\
1.5122\end{array}$ & 1.4953 & 1.4669 & 1.9649 & 1.4901 & 1.7619 & 1.0475 & 1.4886 & 0.6187 & 1.7252 \\
\hline $\begin{array}{l}\text { Operating load } \\
1.8112\end{array}$ & 1.2351 & 1.7676 & 1.9424 & 1.2286 & 1.3787 & 0.6792 & 1.2257 & 1.3806 & 1.8446 \\
\hline $\begin{array}{l}\text { Operating load } \\
2.5122\end{array}$ & 0.5201 & 0.7542 & 0.2733 & 0.5224 & 1.9419 & 0.7901 & 0.5137 & 1.9909 & 1.8075 \\
\hline \multicolumn{10}{|c|}{ Value of GVSM for unknown loading at signal-to-noise ratio $0.05 \mathrm{db}$} \\
\hline & NR & FFBP & $\mathrm{CFB}$ & GR & LR & NARX & RBFN & ELMAN & FFDTD \\
\hline $\begin{array}{l}\text { Operating load } \\
1.1628\end{array}$ & 1.9862 & 2.0004 & 2.0038 & 1.9539 & 0.6863 & 1.7635 & 1.9788 & 0.4354 & 1.6397 \\
\hline $\begin{array}{l}\text { Operating load } \\
1.2118\end{array}$ & 1.8617 & 2.0035 & 2.0231 & 1.8682 & 0.3445 & 1.5947 & 1.8557 & 0.5822 & 1.8311 \\
\hline $\begin{array}{l}\text { Operating load } \\
1.5122\end{array}$ & 1.4953 & 1.8995 & 1.9757 & 1.4905 & 0.8095 & 1.0957 & 1.4789 & 1.6404 & 1.8227 \\
\hline $\begin{array}{l}\text { Operating load } \\
1.8112\end{array}$ & 1.2351 & 0.7702 & 1.7993 & 1.2248 & 1.8876 & 0.8717 & 1.2122 & 1.0576 & 1.8252 \\
\hline $\begin{array}{l}\text { Operating load } \\
2.5122\end{array}$ & 0.5201 & 0.8744 & 0.2835 & 0.5355 & 1.7867 & 0.4752 & 0.512 & 1.9095 & 1.6913 \\
\hline
\end{tabular}

the values of CVI is observed with the increase in operating load.

(2) In case of pi-equivalent models, the variation of CVI is mainly dependent on the values of parameter $A$. The values of " $A$ " parameter are system specific and depend on system's parameters (susceptance and impedance). With the change in operating conditions these values are changed. For IEEE 14-bus system, the values of CVIs are around 0.513 near base load, 0.54 near medium load, and 0.65 at heavy loading. Similarly for IEEE 30-bus system, the values of CVIs are 0.51 for nominal loading, 0.53 for medium loading, and 0.584 for heavy loading. For IEEE 118-bus test system the values of CVIs are comparatively high as compared to the small test systems. For high loading (Case 3) system has reached to the point of collapse. Values of CVIs are nearly equal to 1 in this case. After observing these values it can be concluded that point of collapse is dependent on system parameters.

\section{Conclusion}

The paper proposes an online assessment of voltage stability for multimachine networks with the application of RBFN. An equivalent two-bus pi-network model is developed for assessment of voltage stability for multibus power systems where series and shunt parameters of transmission lines are lumped separately in the form of series and shunt equivalents. GVSM is used to assess the voltage instability or in other words to assess the proximity of the existing system state from voltage collapse. the following are the major highlights of this work.

(a) GVSM for the given power system networks are calculated to judge the health of the power system. 
TABLE 4: GVSM for IEEE 118-bus test system at unknown different operating loading incorporating noise.

\begin{tabular}{|c|c|c|c|c|c|c|c|c|c|}
\hline \multicolumn{10}{|c|}{ Value of GVSM for unknown loading at normal case } \\
\hline & NR & FFBP & CFB & GR & LR & NARX & RBFN & ELMAN & FFDTD \\
\hline $\begin{array}{l}\text { Operating load } \\
1.249\end{array}$ & 5.1187 & 5.0112 & -1.762 & 5.133 & -6.4245 & -1.9971 & 5.118 & 0.2715 & -8.6754 \\
\hline $\begin{array}{l}\text { Operating load } \\
1.478\end{array}$ & 4.0734 & 4.541 & -3.1588 & 4.1287 & -8.4655 & -7.3933 & 4.0729 & 0.1209 & -8.5274 \\
\hline $\begin{array}{l}\text { Operating load } \\
1.5671\end{array}$ & 3.5154 & 3.6765 & -5.303 & 3.5939 & -8.7183 & -8.2413 & 3.515 & 1.0685 & -8.4834 \\
\hline $\begin{array}{l}\text { Operating load } \\
1.6591\end{array}$ & 2.9059 & 1.8745 & -7.2875 & 3.05 & -7.5549 & -8.6219 & 2.9041 & 1.3871 & -8.4485 \\
\hline $\begin{array}{l}\text { Operating load } \\
1.8751\end{array}$ & -4.6571 & -3.8761 & -10.1198 & -3.4453 & -3.2183 & -8.8976 & -4.6565 & 1.4657 & -8.3791 \\
\hline \multicolumn{10}{|c|}{ Value of GVSM for unknown loading at signal-to-noise ratio $0.5 \mathrm{db}$} \\
\hline & NR & FFBP & CFB & GR & LR & NARX & RBFN & ELMAN & FFDTD \\
\hline $\begin{array}{l}\text { Operating load } \\
1.249\end{array}$ & 5.1187 & 5.0187 & 0.2122 & 5.1415 & -10.8296 & -5.02 & 5.105 & -1.3566 & -8.456 \\
\hline $\begin{array}{l}\text { Operating load } \\
1.478\end{array}$ & 4.0734 & 5.2299 & -9.4885 & 4.1286 & -8.0771 & -8.7955 & 4.0235 & -6.1076 & -8.1775 \\
\hline $\begin{array}{l}\text { Operating load } \\
1.5671\end{array}$ & 3.5154 & 5.0041 & -8.5883 & 3.6213 & -5.2684 & -7.7728 & 3.505 & 2.8002 & -7.9819 \\
\hline $\begin{array}{l}\text { Operating load } \\
1.6591\end{array}$ & 2.9059 & 3.7894 & -7.4151 & 3.05 & -1.8476 & -7.779 & 2.8051 & 0.7538 & -8.2134 \\
\hline $\begin{array}{l}\text { Operating load } \\
1.8751\end{array}$ & -4.6571 & -5.0553 & -8.5239 & -3.4719 & -6.0628 & -8.9145 & -4.637 & -0.1573 & -8.5286 \\
\hline \multicolumn{10}{|c|}{ Value of GVSM for unknown loading at signal-to-noise ratio $0.05 \mathrm{db}$} \\
\hline & NR & FFBP & CFB & GR & LR & NARX & RBFN & ELMAN & FFDTD \\
\hline $\begin{array}{l}\text { Operating load } \\
1.249\end{array}$ & 5.1187 & 5.2705 & -0.3736 & 5.133 & -10.638 & -5.9343 & 5.0081 & 1.2566 & -8.2898 \\
\hline $\begin{array}{l}\text { Operating load } \\
1.478\end{array}$ & 4.0734 & 5.2223 & -2.7077 & 4.1287 & -2.5001 & -5.5532 & 4.0101 & -1.3369 & -7.759 \\
\hline $\begin{array}{l}\text { Operating load } \\
1.5671\end{array}$ & 3.5154 & 3.436 & 1.4016 & 3.5934 & -6.853 & -8.663 & 1.5013 & 3.5063 & -8.4106 \\
\hline $\begin{array}{l}\text { Operating load } \\
1.6591\end{array}$ & 2.9059 & 1.9993 & -7.9334 & 3.0947 & -4.1361 & -8.425 & 2.2041 & 2.643 & -8.0591 \\
\hline $\begin{array}{l}\text { Operating load } \\
1.8751\end{array}$ & -4.6571 & -3.9595 & -10.7149 & -3.4397 & -7.2241 & -8.8729 & -4.3073 & 1.4507 & -8.4814 \\
\hline
\end{tabular}

Prediction of GVSM and CVI by different neural network topologies is validated through offline NR method.

(b) The main advantage of the proposed method is that it indicates a good agreement between target data $(\mathrm{N}$ $\mathrm{R}$ ) and RBFN output. Prediction accuracy of RBFN is best as compared with other topologies of ANN.

(c) The proposed approach provides fast computation of GVSM. Operator can analyze severity of any unknown load pattern by using this supervised learning approach.

(d) Prediction accuracy of RBFN is best. It is validated by introduction of AWGN in the system with different levels of SNR.

Application of Support Vector Machine (SVM) in voltage stability assessment lies in the scope of the future work.

\section{Nomenclature}

$R: \quad$ Resistance of the line

$X: \quad$ Reactance of the line

$Z: \quad$ Impedance of the line

$Z_{\text {se_eq }}: \quad$ Equivalent series impedance

$Z_{\text {sh_eq }}: \quad$ Equivalent shunt impedance

$V_{\mathrm{s}}, V_{\mathrm{r}}$ : Voltage at sending bus and receiving bus, respectively

$I_{\mathrm{s}}, I_{\mathrm{r}}$ : Current at sending bus and receiving bus, respectively

$I_{\text {se }}$ : Current through series equivalent impedance

$I_{\text {shs }}, I_{\text {shr }}$ : Shunt branch currents at sending and receiving end sides, respectively

$V_{\mathrm{cr}}: \quad$ Critical voltage index

$S_{\mathrm{g}}$ : $\quad$ Apparent power of generator

$S_{\text {load }}$ : Apparent power at load end side 
$P_{\mathrm{g}}, Q_{\mathrm{g}}: \quad$ Active and reactive power of generator, respectively

$P_{\mathrm{r}}, Q_{\mathrm{r}}$ : $\quad$ Active and reactive power at load end side, respectively

$m, n: \quad$ Nodes

$Q_{2}$ : $\quad$ Reactive power at receiving bus

"s" and "r": Symbols for sending and receiving side, respectively

$\left[\begin{array}{ll}A & B \\ C & D\end{array}\right]: \quad$ Transmission $(A B C D)$ parameters

$\delta: \quad$ Voltage angle

$\alpha: \quad$ Angle of magnitude of parameter $A$

$\beta$ : $\quad$ Angle of magnitude of parameter $B$

$\theta$ : $\quad$ Angle of receiving end voltage $V_{\mathrm{s}}$

$\Delta[J]: \quad$ Determinant of Jacobian matrix

SNR: $\quad$ Signal-to-noise ratio

N-R: Newton Raphson

GVSM: Global Voltage Stability Margin

MLP: $\quad$ Multilayer Perceptron

LM: Levenberg-Marquardt

AWGN: Additive White Gaussian Noise

RBFN: Radial Basis Function Network

FFBP: $\quad$ Feedforward Backprop

CFB: $\quad$ Cascade Forward Backprop

GR: Generalized Regression

LR: $\quad$ Layer Recurrent

FFDTDN: Feedforward Distributed Time Delay Network.

\section{Competing Interests}

The authors declare that they have no competing interests.

\section{Acknowledgments}

The authors acknowledge the support and encouragement of Swami Keshvanand Institute of Technology, Management \& Gramothan, Jaipur, Rajasthan, India. The authors also would like to thank Ms. Neha Purohit, Reader, Department of English, Swami Keshvanand Institute of Technology, Management \& Gramothan, for valuable suggestions.

\section{References}

[1] P. Kundur, J. Paserba, V. Ajjarapu et al., "Definition and classification of power system stability," IEEE Transactions on Power Systems, vol. 19, no. 3, pp. 1387-1401, 2004.

[2] P. Kundur, Power System Stability and Control, McGraw-Hill, New York, NY, USA, 1994.

[3] P. Nagendra, S. H. nee Dey, and S. Paul, "An innovative technique to evaluate network equivalent for voltage stability assessment in a widespread sub-grid system," International Journal of Electrical Power \& Energy Systems, vol. 33, no. 3, pp. 737-744, 2011.

[4] P. Kessel and H. Glavitsch, "Estimating the voltage stability of a power system," IEEE Transactions on Power Delivery, vol. 1, no. 3, pp. 346-354, 1986.

[5] F. Gubina and B. Strmcnik, "Voltage collapse proximity index determination using voltage phasors approach," IEEE Transactions on Power Systems, vol. 10, no. 2, pp. 788-794, 1995.
[6] Y. Wang, W. Li, and J. Lu, "A new node voltage stability index based on local voltage phasors," Electric Power Systems Research, vol. 79, no. 1, pp. 265-271, 2009.

[7] S. Dey, C. K. Chanda, and A. Chakrabarti, "Development of a global voltage security indicator (VSI) and role of SVC on it in longitudinal power supply (LPS) system," Electric Power Systems Research, vol. 68, no. 1, pp. 1-9, 2004.

[8] R. Tiwari, K. R. Niazi, and V. Gupta, "Line collapse proximity index for prediction of voltage collapse in power systems," International Journal of Electrical Power and Energy Systems, vol. 41, no. 1, pp. 105-111, 2012.

[9] G. B. Jasmon, L. H. Callistus, and C. Lee, "Prediction of voltage collapse in power systems using a reduced system model," in Proceedings of the IEE International Conference on Control, pp. 32-36, London, UK, March 1991.

[10] H. G. Kwatny, A. K. Pasrija, and L. Y. Bahar, "Static bifurcations in electric power networks: loss of steady-state stability and voltage collapse," IEEE Transactions on Circuits and Systems, vol. 33, no. 10, pp. 981-991, 1986.

[11] V. Ajjarapu and B. Lee, "Bifurcation theory and its application to nonlinear dynamical phenomena in an electrical power system," IEEE Transactions on Power Systems, vol. 7, no. 1, pp. 424-431, 1992.

[12] B. Gao, G. K. Morison, and P. Kundur, "Voltage stability evaluation using modal analysis," IEEE Transactions on Power Systems, vol. 7, no. 4, pp. 1529-1542, 1992.

[13] C. A. Canizares, A. C. Z. De Souza, and V. H. Quintana, "Comparison of performance indices for detection of proximity to voltage collapse," IEEE Transactions on Power Systems, vol. 11, no. 3, pp. 1441-1450, 1996.

[14] C. A. Canizares, "On bifurcations, voltage collapse and load modeling," IEEE Transactions on Power Systems, vol. 10, no. 1, pp. 512-522, 1995.

[15] S. Konar, D. Chatterjee, and S. Patra, "V-Q sensitivity-based index for assessment of dynamic voltage stability of power systems," IET Generation, Transmission \& Distribution, vol. 9, no. 7, pp. 677-685, 2015.

[16] A. K. Sharma, A. Saxena, and R. Tiwari, "Voltage stability assessment using GVSM and preventive control using SVC," International Journal of Computer Applications, vol. 142, no. 11, pp. 23-31, 2016.

[17] M. T. Hagan, H. B. Demuth, and M. H. Beale, Neural Network Design, 2nd edition, 1995.

[18] http://www.mathworks.com.

[19] Power System Test Archive-UWEE, University of Washington, https://www.ee.washington.edu/research/pstca. 


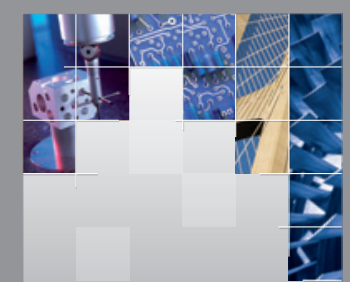

\section{Enfincering}
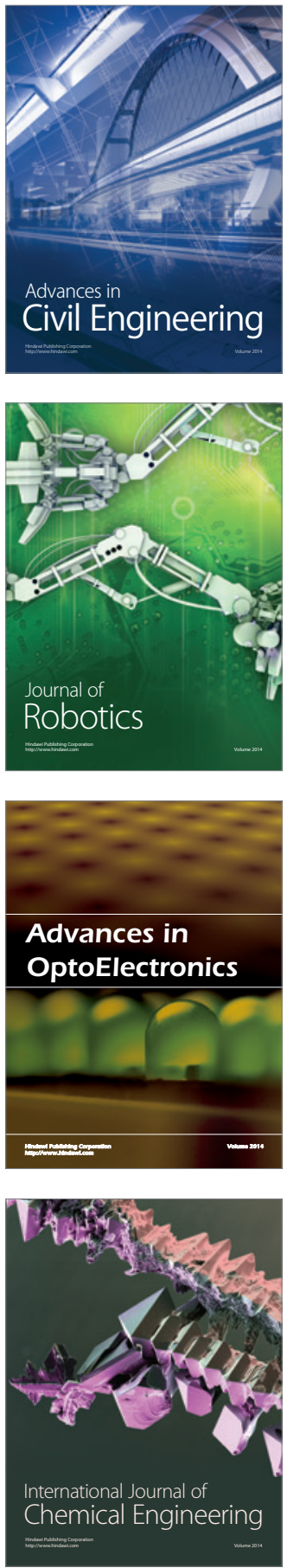

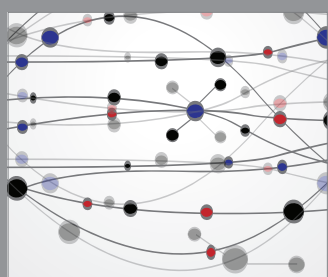

The Scientific World Journal

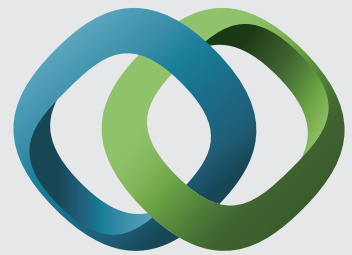

\section{Hindawi}

Submit your manuscripts at

http://www.hindawi.com
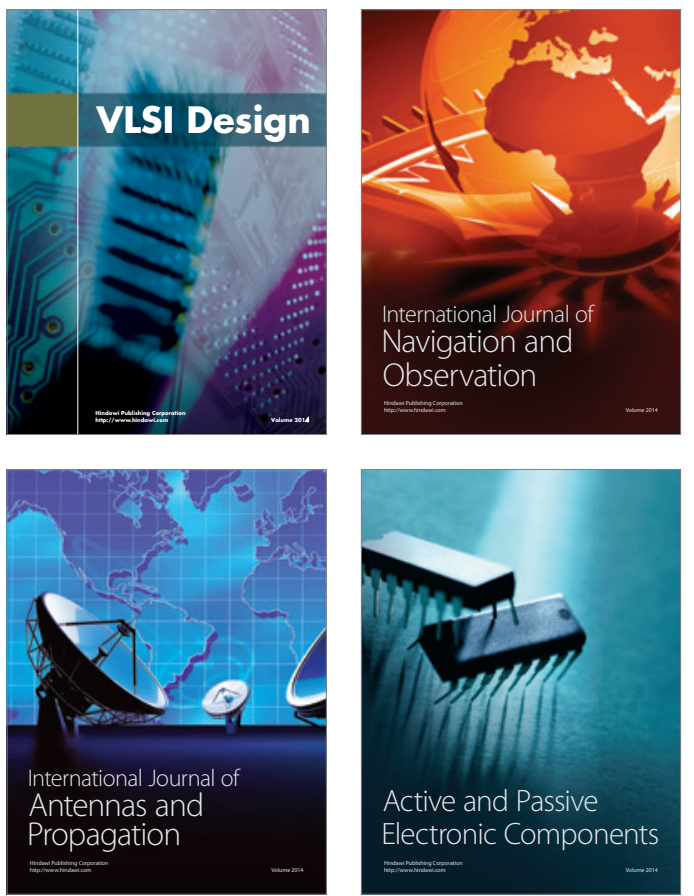
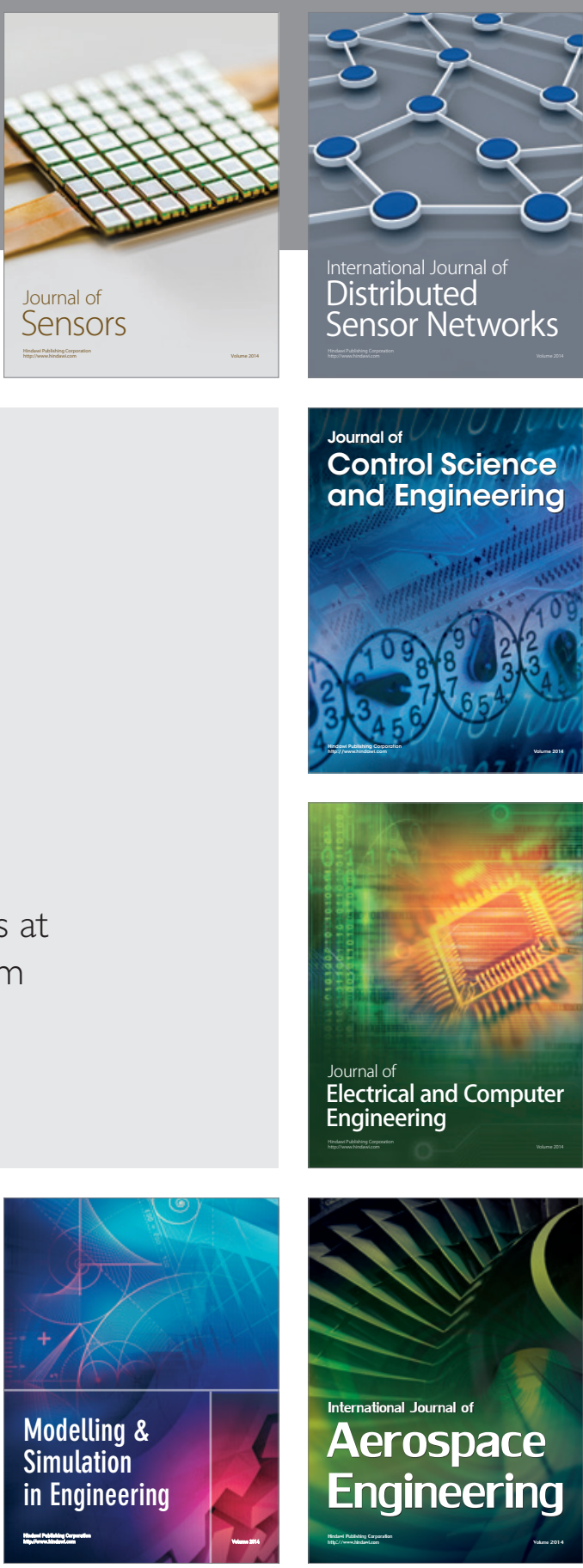

International Journal of

Distributed

Sensor Networks

Journal of

Control Science

and Engineering
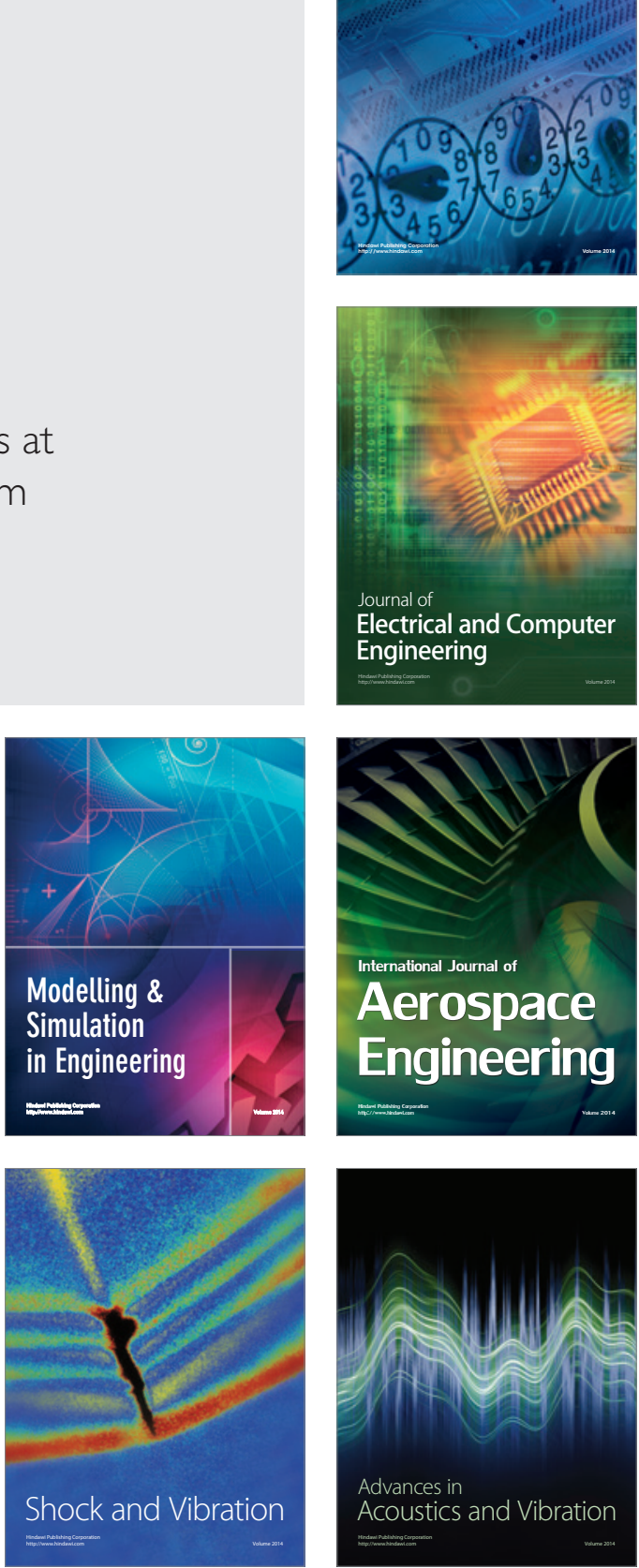